The intake was adjusted for within-person variability by the method proposed by lowa State University, using the software PCSIDE, and energy-adjusted for the residual method. The validity for 23 nutrients was assessed by Spearman correlation coefficient and weighted $\kappa$. Intra-class correlation coefficient and weighted $\kappa$ was used to reproducibility assessment.

Results In the validation, the Spearman correlation coefficients ranged from 0.25 (riboflavin) to 0.57 (phosphorus) of which eight nutrients showed acceptable correlation $(>0.4)$. In the reproducibility analysis, the intra-class correlation coefficients ranged from 0.18 (vitamin C) to 0.57 (niacin), of which five nutrients showed acceptable correlation. The weighted $\kappa$ ranged from 0.18 (sodium) to 0.67 (riboflavin) to reproducibility and from 0.19 (polyunsatured fat) to 0.56 (calcium) to validation.

Conclusion The results support the use of this instrument to assess food intake in epidemiological studies conducted in São Paulo, Brazil.

\section{SP3-79 AUDIT OF A NATIONWIDE PATHOLOGY-BASED CANCER REGISTRY IN IRAN AND LESSONS LEARNED FROM SUCCESSFUL POPULATION-BASED CANCER REGISTRIES WORLDWIDE}

doi:10.1136/jech.2011.1429760.79

K Zendehdel, G Keshtmand, * Z Sedighi, J Hassanloo, A Nahvijou, M A Mohagheghi. Cancer Research Center, Cancer Institute of Iran, Tehran University of Medical Sciences, Tehran, Iran

Background Several low- and middle- income countries, lack wellfunctioning population-based cancer registry. We evaluated the completeness of a pathology based cancer registry in Iran. We further studies evolutionary progress of the cancer registries worldwide.

Methods We evaluated consistency of the incidence rates reported by national pathology-based cancer registry in Iran from 2004 to 2007. We further compared the incidence rates of the pathology- and population-based registries in a few regions, where both data were available. In addition, we studied the increasing trend in the number of population-based cancer registries worldwide, using the reports published in the Volumes I-IX of the monograph "Cancer in Five Continents."

Results The Iranian pathology-based cancer registry, reports only about $60-70 \%$ of cancers. The underestimates were greater in cancers with poor-prognosis including lung, stomach, and oesophageal cancers. Almost four regional cancer registries were established every 10 years since 1960. However, the USA was an exception, where the number of cancer registries increased from 14 in 1998 to 44 regional registries in 2002, due to the advance infrastructure in the health informatics and ambitious initiatives by the Centers for Disease Control in the USA.

Conclusions Pathology based cancer registry cannot provide reliable estimate for the cancer incidence rates, particularly in cancers with a poor prognosis. Developing countries should establish and support regional registries and expand their coverage gradually. Given the recent advances in the health informatics, small efforts will enhance the coverage of cancer registries worldwide, particularly in the less than middle income countries.

\section{SP3-80 PARENT'S SOCIAL STATUS AND EARLY NUTRITION INFLUENCES ON COLLEGE ENTRANCE AMIONG BRAZILIAN YOUTH AT 2 DECADES}

doi:10.1136/jech.2011.1429760.80

L Baraldi, ${ }^{*}$ W Conde. Public Health School, São Paulo, São Paulo, Brazil

Introduction Human capital concept is related to social and nutrition experiences in childhood as well as social status inherited from their parents.
Objective Estimate the chance of university achievement among Brazilian youth with adequate or inadequate early nutrition and born from parents with low vs high school degree.

Methods A sample of young, aged 20-24 years, and their parents from three Brazilian surveys, PNSN-1989, POF-2003 and POF-2009. Nutritional status was standardised from WHO reference2007; highest school level achieved was used to classify social status. We set three nutrition groups (below $-1 Z,-1$ to +1 , and above $+1 Z$, named N1, N2 and N3, respectively) for parents and youth and three school levels groups (elementary, high, college, named E1, E2, E3 respectively) for parents. Probability of beginning university by youth was estimated using multiple logistic regression. Survey year was included as independent dummy variable to estimate changes among periods.

Results The ratio between parents E3 vs E1 among youth N1 was 7.0 in 1989, 11.0 in 2003 and 6.1 in 2009. This ratio for young N2 was 5.9 in 1989, 9.7 and 5.0 in 2003 and 2009, respectively. For young's N3 that ratio was 3.9 in 1989, 8.8 in 2003 and 4.0 in 2009. Conclusion The unequal chance of being university student in Brazil as function of parent's social status and early nutrition decreased from period 1989-2003 to period 2003-2009. Probably this change is associated with social mobility experienced in country this decade.

\section{SP3-81 GEO-EPIDEMIOLOGY OF MULTIDRUG-RESISTANT TUBERCULOSIS CASES IN THE STATE OF SÃO PAULO, BRAZIL, 2007-2010}

doi:10.1136/jech.2011.1429760.81

P A Opromolla, ${ }^{*}$ V M Galesi, L A R dos Santos, M J P Rujula. Secretaria de Estado da Saúde, São Paulo, São Paulo, Brazil

In São Paulo, resistant tuberculosis (MDR-TB) is defined for the purpose of notification and treatment, as those cases that present with in-vitro resistance to rifampicin and isoniazid, and a third standard drug. There is currently a system of epidemiological surveillance of MDR-TB, which monitors all re-treatment cases of the disease nationwide. In the State of São Paulo, this monitoring is done by the division of tuberculosis Epidemiological Surveillance Centre of São Paulo. The aim of this study was to analyse the spatial distribution of cases of resistant tuberculosis in the state of São Paulo, Brazil, in the period 2007-2010.

Methods We selected confirmed cases of MDR-TB, notified to the state of Sao Paulo in the period 1 January 2007 to 10 January 2011 with the county as the unit of spatial analysis. We also analysed data from incident cases of tuberculosis in the state during the same period and with the same spatial unit of analysis. In addition to these data, information about detention facilities was also collected. All data were analysed in GIS, using space techniques for the detection of clusters and spatial correlations.

Results We detected 355 cases of MDR-TB during the study period, $34 \%$ in $2010,68.7 \%$ male, $63 \%$ Caucasian and $77 \%$ by 11 years of study. The mean age was 38 years ( \pm 12 years). $97 \%$ were of pulmonary disease, only $11 \%$ were HIV-positive. Using kernel parameters, the greatest probability of MDR-TB cases are concentrated in the southeastern state.

\section{SP3-82 FACTORS ASSOCIATED WITH ORAL HEALTH STATUS OF ADULTS, BRAZIL}

doi:10.1136/jech.2011.1429760.82

${ }^{1} \mathrm{~F}$ Floriano, ${ }^{*} \mathrm{C} C$ Costa Mda, ${ }^{2} \mathrm{M}$ I Vianna, ${ }^{2} \mathrm{M}$ B Cabral, ${ }^{2} \mathrm{M}$ dos Santos, ${ }^{1} \mathrm{G}$ Santos. ${ }^{1}$ ISC-UFBA, SSA, Bahia, Brazil; ${ }^{2}$ FO-UFBA, SSA, Bahia, Brazil

Introduction The objective of this study was to evaluate the oral health status of adults in Salvador-Bahia, Brazil, and to identify 
potentials factors related to tooth loss and loss of periodontal attachment.

Methods Cross-sectional study in 501 adults between 35 and 59 years, living in five different areas covered by the Community Health Agents Program/Family Health Strategy (CHAP/FHS), from July to October 2008. The oral health status information was obtained using indexes and criteria according Examiner's Manual SB Brazil project (2001). Descriptive and exploratory analyses were carried out and the differences in scores were tested by Pearson's $\chi^{2}$.

Results Near $50.0 \%$ of adults had tooth loss $(<20$ functional teeth present) and $17.7 \%$ had loss of periodontal attachment $\geq 6 \mathrm{~mm}$. Female had a greater tooth loss $(53.0 \%)$ and male had larger loss of periodontal attachment $\geq 6 \mathrm{~mm}$ (32.2\%). The adults with more than 45 years presented bigger tooth loss (67.2\%) as much as periodontal attachment $\geq 6 \mathrm{~mm}$ (24.3\%). Among the factors associated with tooth loss, can be pointed out no monthly individual income, low educational level and attending dentist when there is need $(p<0.001)$, while only low educational level was associated with loss of periodontal attachment $\geq 6 \mathrm{~mm}$ $(\mathrm{p}<0.001)$.

Conclusions The oral health status of adults was poor, which makes it one of the priorities of the primary care. Low socioeconomic status is commonly related to this condition, suggesting that improving the socioeconomic determinants reflect improvements in oral health.

\section{SP3-83 RELATIONSHIP BETWEEN CLINICAL DENTAL STATUS AND THE PERCEPTION OF ORAL HEALTH-RELATED QUALITY OF LIFE IN ADULTS, BRAZIL}

doi:10.1136/jech.2011.1429760.83

${ }^{1} \mathrm{~F}$ Floriano, ${ }^{*} \mathrm{C}$ Costa Mda, ${ }^{2} \mathrm{~A}$ Sheiham, ${ }^{2} \mathrm{G}$ Tsakos, ${ }^{3} \mathrm{M}$ I Vianna, ${ }^{3} \mathrm{M}$ B Cabral, ${ }^{1} \mathrm{G}$ Santos, ${ }^{3} \mathrm{M}$ dos Santos. ${ }^{1}$ ISC-UFBA, SSA, Bahia, Brazil; ${ }^{2}$ UCL, London, UK; ${ }^{3} \mathrm{FO}-$ UFBA, SSA, Bahia, Brazil

Introduction The objective of this study was to assess the impact of oral health-related quality of life (OHRQoL) in adults and to investigate the relationship between OHRQoL indicator and specific clinical dental measures.

Methods Cross-sectional survey was carried out of 501 adults between 35 and 59 years, living in five different areas covered by the Community Health Agents Program/Family Health Strategy (CHAP/FHS) in Salvador-Bahia, Brazil. Data were collected from July to October, 2008, through interviews and clinical examination that's used indexes and criteria according Examiner's Manual SB Brazil project (2001). OHRQoL was assessed through the General Oral Health Assessment Index (GOHAI). Data analysis used nonparametric tests (Mann-Whitney, Kruskal-Wallis and multiple linear regressions).

Results $51.1 \%$ of adults had tooth loss ( $<20$ functional teeth present), the GOHAI mean overall score was $29.4 \pm 4.7$ and $50.7 \%$ reported low levels of OHRQoL Female perceived a greater impact of oral health status in quality of life (GOHAI $=29.1 \pm 4.9)$. Concerns about oral health was the most frequently reported problem $(64.9 \%)$ while concerns about appearance $(53.7 \%)$ and oral impacts related to pain $(53.1 \%)$ were experienced by just over than half of participants. Tooth loss remained associated with worse perceptions of OHRQoL $(p<0.05)$ even after adjusted for age, gender, economic status, and loss of periodontal attachment.

Conclusions Poor oral health status increased the perceived impact of OHRQoL. Policies of reduction of oral health inequalities can be successful strategies to improve the quality of life of adults.
SP3-84 IMPACT OF ORAL HEALTH STATUS ON OUALITY OF LIFE IN ADULTS, BRAZIL

doi:10.1136/jech.2011.1429760.84

${ }^{1} \mathrm{~F}$ Floriano, ${ }^{*} \mathrm{C}$ Costa Mda, ${ }^{2} \mathrm{G}$ Tsakos, ${ }^{2} \mathrm{~A}$ Sheiham, ${ }^{3} \mathrm{M}$ I Vianna, ${ }^{3} \mathrm{M}$ B Cabral, ${ }^{3} \mathrm{M}$ dos

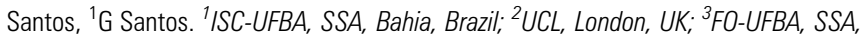
Bahia, Brazil

Introduction The objective of this study was to assess the prevalence of impacts of oral health status on quality of life among adults, Brazil. Methods A cross-sectional survey was carried out of 501 adults between 35 and 59 years, living in five different areas covered by the Community Health Agents Program/Family Health Strategy (CHAP/FHS) in Salvador-Bahia, Brazil. Data were collected from July to October, 2008, through interviews and clinical examination that's used indexes and criteria according Examiner's Manual SB Brazil project (2001). Data on the impact of oral health on the quality of daily living based upon the Oral Impacts on Daily Performance (OIDP) indicator. Exploratory analyses were tested by Pearson's $\chi^{2}$.

Results OIDP mean score was $12.1 \pm 6.3$ and $39.7 \%$ reported that their mouth affected their pattern of daily living. Oral impacts levels were lowest in subjects with the less loss of periodontal attachment. The most common oral impacts were on eating (20.6\%) followed by the ones related to aesthetics (smiling -16.4\%). Approximately 15\% of the adults had oral impacts on cleaning teeth and relaxing. Oral impacts were more prevalent among the female (19.3\%).

Conclusions This survey has shown that the oral status of adults fairly frequently affects their quality of life, and in particular, the ability to eat. Assess the oral health-related Quality of life promotes a more complex understanding of oral health, adds a subjective perspective to clinical interventions that's provide measures to improve the quality of life of patients.

\section{SP3-85 DETERMINANTS OF LIFETIME DENTAL PAIN IN TWO BRAZILIAN BIRTH COHORTS}

doi:10.1136/jech.2011.1429760.85

${ }^{1} \mathrm{M}$ Saraiva, ${ }^{1} \mathrm{H}$ Bettiol, ${ }^{2} \mathrm{~A}$ Silva, ${ }^{1} \mathrm{~T}$ Taglieri, ${ }^{1} \mathrm{~L}$ Taboga, ${ }^{1} \mathrm{M}$ A Barbieri. ${ }^{1}$ University of São Paulo, Ribeirão Preto, Brazil; ${ }^{2}$ Federal University of Maranhão, São Luiz, Brazil

Introduction Dental pain significantly impacts child's life. This study explores the determinants of lifetime dental pain (LDP) among children.

Methods Data from a follow-up of two birth cohorts in Brazil was used: Ribeirão Preto (RP) with one of highest Human Development Index (HDI) in the country and Sao Luiz (SL) one of the lowest. Follow-up data (1/3 of original cohort) was collected in 2004/5 when children from RP were 10/11 years old ( $n=790)$, and children from SL were $7 / 8$ years old $(n=673)$. Prevalence Ratios (PR) were calculated and multivariable analyzes was performed hierarchical modelling. Covariates were mother education, age, marital status, sex, skin colour, number of siblings and number of household members, mother's perception of child oral health, dental visit, and private dental insurance.

Results LDP prevalence in RP was $32.7 \%$, while in SL was $56.4 \%$. Common variables in the final model for the two cities were sex, mother's education, mother's perception of child oral health. For RP private health insurance (PR 0.67; 95\% CI 0.46 to 0.95 ) and marital status (PR 1.10; 95\% CI 0.87 to 1.40 ) were also included in the model. For SL visiting dentist in the past 2 years was positively associated with LDP (PR: 1.22 95\% CI 1.07 to 1.39 ) as well as the number of household members with children in families with more then five members showing PR 1.36 (95\% CI 1.03 to 1.79).

Conclusion The prevalence of LDP in both cohorts was relatively high and specially associated with family structure and socioeconomic position. 